\title{
SCENARIO-BASED GENERATION OF ONTOLOGIES FOR DOMAIN-SPECIFIC LANGUAGES
}

\author{
Bharvi Chhaya \\ Shafagh Jafer \\ Department of Electrical, Computer, Software \\ and Systems Engineering \\ Embry-Riddle Aeronautical University \\ 1 Aerospace Boulevard \\ Daytona Beach, FL, USA \\ chhayab@my.erau.edu, jafers@erau.edu
}

\begin{abstract}
Ontology-Driven Software Development has emerged as a significant mechanism in creating domainspecific languages (DSLs), allowing for expressing domain concepts effectively. Once an ontology is built, it is a simple process to generate the language's metamodel and establish relationships among related concepts. However, the creation of the ontology is often a time-consuming and laborious task requiring familiarity with the domain being modeled as well as a basic understanding of ontology engineering. This paper discusses the use of natural language scenarios to create a domain ontology, which can be applied towards generating a metamodel for a DSL. A case study for aviation scenarios is presented by using increasing numbers of scenarios and comparing the models at different stages to observe the evolution of the ontology. The scenario-driven ontology developed is compared to an existing aviation ontology to understand its value.
\end{abstract}

Keywords: domain-specific languages, ontologies, scenarios, scenario-driven development.

\section{INTRODUCTION}

The use of domain-specific languages (DSLs) has increased manifold for problem-solving in specific domain areas as they allow for a wider variety of expressions within their domain. Modeling using DSLs has shown high increases in productivity after accounting for the time and cost expended in developing them, making them a suitable target for improvement in order to reap higher rewards (Thibault, Marlet and Consel 1999). DSL development is incredibly difficult as it requires knowledge of the domain and language development expertise, and usually few people have both. As a result, the creation of a DSL is often not considered and if so, a DSL rarely evolves into a full-fledged language which can be used within the domain it is intended for (Mernik, Heering and Sloane 2005).

The currently used approach for domain modeling involves the creation of an ontology which is then used to describe the domain model (Pereira, Fonseca and Henriques 2016). This ontology encapsulates all domain knowledge and can be cumbersome to create, requiring external sources of information and assistance from a domain expert (Čeh, et al. 2011).

An ontology describes the concepts and relationships that are important in a particular domain and provides a vocabulary for that domain (Yao and Zhang 2009). An ontology provides a quick and simplified description of a DSL, abstracting language's technical details, while highlighting key terminology and 
specifics. Once an ontology is built, it is a simple process to generate the language's metamodel and establish relationships among related concepts. An ontology-based approach to DSL modeling requires the properties of the entire domain to be captured within an ontology prior to the start of modeling.

Among existing ontology specification frameworks, the Web Ontology Language format (OWL) (Bechhofer 2009) is most commonly used by the DSL community. OWL describes a domain in terms of classes, properties and individuals and may include rich descriptions of the characteristics of those objects (Bechhofer 2009). Protégé (Protégé Home Page 2016) is a popular tool used for generating OWL ontologies (Alatrish 2013).

In the field of modeling and simulation (M\&S), DSLs are generally used to create scenarios for simulations (Jafer, Chhaya and Durak, et al. 2018). The term "scenario" has a variety of published definitions from a vast array of domains. While the term itself has varying meanings, two definitions represent a scenario in the context of simulation development and usage. First, a scenario can be defined as a description of the hypothetical or real area, environment, means, objectives, and events during a specified time frame related to events of interest (GSD Product Development Group, SISO 2014). Second, a scenario can be defined as a specification of conditions and situations to be represented by a simulation environment for its purpose (Durak, et al. 2014). Both definitions agree that a scenario is a description of important events and conditions needed to represent a specific order of events which, in this application, occurs within a simulation environment. Therefore, when developing a simulation environment which utilizes these events and conditions, it is often a prerequisite to define the scenarios that will be executed in the target simulation environment.

Each scenario needs to specify three main components in order to be considered complete: (1) the initial state, (2) the course of events, and (3) the termination conditions. As the scenario development process progresses, these three components should be further refined and expanded. The initial state describes the situation at the beginning of the scenario timeline and generally contains information such as date and time, surrounding conditions, and objects. Any number of events can occur throughout the scenario timeline. The termination conditions describe the state of the simulation environment where the scenario can be defined as completed or terminated. This can occur via a specific event (such as a successful landing) or other means of measurement (such as achieving a predefined elapsed time) (GSD Product Development Group, SISO 2014).

Scenario development can be broken down into the creation of three scenario groups: (1) operational scenarios, (2) conceptual scenarios, and (3) executable scenarios. Operational scenarios are provided by subject-matter experts (SMEs) in the early stages of development. These scenarios often provide a broad description of the desired events in textual form using natural language. For example, an operational scenario may describe what events occur during the simulation and in which order they should occur. A conceptual scenario adds detail and additional information to the operational scenarios. These scenarios are often created by an M\&S expert in collaboration with an SME. Although similar to an operational scenario, conceptual scenarios contain all information needed for the simulation environment. Finally, once the conceptual model is complete and the simulation environment is defined, executable scenarios can be made. An executable scenario is the specification of a specific situation providing all information necessary for preparation, initialization, and execution of a simulation environment (GSD Product Development Group, SISO 2014, Durak, et al. 2014).

This paper discusses operational scenarios which are already provided at the beginning of the modeling process and how they could be used to create a domain ontology. This approach proposes the use of scenarios in natural language, which are currently used in requirements engineering and testing, as the basis for developing an ontology for the domain model iteratively.

There have been some published works on the use of natural-language texts for ontology learning (Shamsfard and Barforoush 2003). Two such projects are ASIUM and Hasti. ASIUM (Faure, Nédellec and Rouveirol 1998) provides a methodology for learning verb frames and taxonomic knowledge, based on 
statistical analysis of syntactic parsing of French texts. Hasti (Shamsfard and Barforoush 2004) is an extensive model for ontology learning from scratch. Hasti extracts lexical and ontological knowledge from Persian (Farsi) texts. While the idea of the proposed approach is the same in the use of natural language text to build an ontology, the type and application is more specific. ASIUM and Hasti incorporate the knowledge of the entire domain through learning from extensive texts. The approach suggested here can be used to build an ontology for domain modeling using a much smaller body of input, which is the operational scenarios provided at the beginning of the modeling process.

\section{USING SCENARIOS TO GENERATE ONTOLOGIES}

\subsection{Methodology}

This approach aims to use natural language scenarios to extract key terminology in the domain with the assumption that all key concept would appear regularly in natural-language scenario descriptions. This approaches analyzes word frequency in operational scenarios to distinguish these important keywords. It is presumed that articles and prepositions can be ignored when looking for domain-specific keywords. Verbs can be used to determine what entities interact with each other and how within a simulation, and nouns are the chief elements that are specific to the domain. In order to obtain an accurate domain model, a large number of scenarios needs to be analyzed and the evolution of the model with addition of scenarios needs to be observed. The case study in this section will use flight training scenarios to obtain an ontology for aviation, currently specific to a flight simulation.

\subsection{Single Scenario Text}

First, the text of a single scenario is parsed to see if any words stand out when checking for frequency. The scenario used here has been obtained from the Federal Aviation Administration (FAA) example scenarios for flight instructors (Federal Aviation Administration 2013). The first scenario chosen is:

In this scenario, the flight starts out as a VFR flight to a nearby airport. Along the way, put the student instructor put on a view limiting device and demonstrate each of the basic attitude instrument maneuvers. At some point, put the student into an unusual attitude and demonstrate the proper recovery procedures.

Upon arrival at the destination airport, have the student instructor demonstrate a slip to a landing. While in the traffic pattern, have the student instructor demonstrate a go around from a rejected landing while explaining the key elements that go into deciding when to reject a landing.

At this point, you can remain in the traffic pattern and complete another landing or depart the pattern for the return trip depending on how the student instructor performs the approach and landings.

During the return flight, the student instructor should have you put on a view limiting device. For safety purposes, you can simulate the role of a student wearing a view limiting device. The student instructor should be able to properly explain each of the maneuvers to be flown as well as properly evaluate your performance on each one you demonstrate. During this time, you can help the student instructor learn proper techniques for putting students into unusual attitudes without imposing undue stress on the airplanes or putting the flight at risk.

Word frequency analysis shows that there are 13 words in this scenario that appear at least four times in it, as shown in

Table 1. After removing articles and other parts of speech, we are left with only four nouns that can be considered part of the domain: student, instructor, flight and landing. All words in the scenario were considered in this case. Using a single scenario with 104 unique words, we have been able to distinguish four concepts relevant to pilot training and we only have to establish the relationships between them. 
Table 1: Frequency of words obtained from first scenario.

\begin{tabular}{|l|l|l|}
\hline Word(s) & Occurrence & Percentage \\
\hline The & 24 & $10.71 \%$ \\
\hline A & 11 & $4.90 \%$ \\
\hline Student & 9 & $4.02 \%$ \\
\hline Instructor & 7 & $3.13 \%$ \\
\hline On, to, you, demonstrate & 5 & $2.32 \%$ \\
\hline At, flight, and, put, landing & 4 & $1.79 \%$ \\
\hline
\end{tabular}

\subsection{Addition of other scenarios from the same source}

Clearly, \{student, instructor, flight, landing\} are important concepts in flight training simulation, but they cannot describe a full scenario. In order to find more keywords, other scenarios have to be used. In this section, 10 more scenarios were used from the same source (Federal Aviation Administration 2013) in order to have key concepts that are described similarly. Once these were analyzed, 439 unique words were found. An occurrence threshold of minimum five times in the 10 scenarios was imposed in order to consider the important domain concepts. Only nouns have been included in the model at this time and hence shown in Table 2 in order to save space.

Table 2: Frequency of nouns obtained from 10 scenarios.

\begin{tabular}{|l|l|l|}
\hline Word(s) & Occurrence & Percentage \\
\hline Student & 89 & $3.82 \%$ \\
\hline Instructor & 73 & $3.13 \%$ \\
\hline Flight & 30 & $1.29 \%$ \\
\hline Approach & 28 & $1.20 \%$ \\
\hline Airport & 20 & $0.86 \%$ \\
\hline Landing, instrument & 14 & $0.60 \%$ \\
\hline Scenario, runway & 10 & $0.43 \%$ \\
\hline Airplane, pattern & 7 & $0.30 \%$ \\
\hline Emergency, traffic, takeoff & 6 & $0.26 \%$ \\
\hline Engine, ground & 5 & $0.21 \%$ \\
\hline
\end{tabular}

As can be seen from the table, the domain keywords begin to take shape once additional scenarios are added. As the sample size in this case was smaller, words that are repeated up to 5 times were chosen; however, the threshold is much larger when there is a longer list of available scenarios. 


\subsection{Addition of other scenarios from different sources}

Once several keywords were identified, scenarios from different sources were chosen in order to maximize the content of the domain covered. After adding 20 scenarios from Calfior and Miller (1994) to the list of 10 scenarios by the FAA (2013), the frequency of words was analyzed again to obtain the nouns shown in Table 3. This time, there were 1092 unique words, of which 120 appeared 10 times or more in the text. The occurrence threshold of minimum 10 times in the 20 scenarios was imposed in order to consider the important domain concepts.

Table 3: Frequency of nouns obtained from 30 scenarios from three sources.

\begin{tabular}{|l|l|l|}
\hline Word(s) & Occurrence & Percentage \\
\hline Student & 89 & $1.29 \%$ \\
\hline Approach & 81 & $1.17 \%$ \\
\hline Instructor, airport & 73 & $1.06 \%$ \\
\hline Flight, runway, departure & 56 & $0.81 \%$ \\
\hline Landing, climb, turn & 34 & $0.49 \%$ \\
\hline Altitude, descent, cruise, pattern & 21 & $0.30 \%$ \\
\hline Instrument & 19 & $0.28 \%$. \\
\hline Route, point, control & 14 & $0.20 \%$ \\
\hline Enroute, fix, IFR & 12 & $0.17 \%$ \\
\hline Fuel, scenario & 11 & $0.16 \%$ \\
\hline Land, traffic, takeoff & 10 & $0.14 \%$ \\
\hline
\end{tabular}

\section{GENERATING ONTOLOGY FROM IDENTIFIED KEYWORDS}

The 30 scenarios yielded 26 unique elements for the domain. This is a very small number and can be adjusted by changing the repetition threshold in the number of occurrences or by analyzing more scenarios. However, as this is a case study, the number is considered sufficient.

\subsection{Relationships between elements}

Now that keywords have been identified, the domain only needs to be studied with respect to these elements in order to understand the relationships between them. Through a cursory review of the 26 keywords in aviation, the hierarchical relationships of these elements are shown in Table 4. The words in angular brackets, i.e. $<$ text $>$, were not originally part of the scenarios, but were discovered when studying the domain for the nouns obtained.

Table 4: Relationships between elements identified from scenarios.

\begin{tabular}{|l|l|}
\hline Parent Element & Components \\
\hline$<$ Pilot $>$ & Student \\
\cline { 2 - 2 } & Instructor \\
\hline
\end{tabular}


Chhaya, and Jafer

\begin{tabular}{|l|l|}
\hline Airport & Runway \\
\hline Flight & Fuel \\
\cline { 2 - 2 }$<$ Flight State $>$ & IFR \\
\cline { 2 - 2 } & Route \\
\cline { 2 - 2 } & Altitude \\
\hline \multirow{5}{*}{} & Approach \\
\cline { 2 - 2 } & Climb \\
\cline { 2 - 2 } & Turn \\
\cline { 2 - 2 } & Descent \\
\cline { 2 - 2 } & Cruise \\
\cline { 2 - 2 } & Enroute \\
\cline { 2 - 2 } & Pattern \\
\cline { 2 - 2 } & Departure \\
\cline { 2 - 2 } & Landing \\
\cline { 2 - 2 } & Takeoff \\
\hline$<$ Air $>$ Traffic Control & \\
\hline$<$ Airspace $>$ & Fix \\
\cline { 2 - 2 } & Point \\
\hline Scenario & \\
\hline
\end{tabular}

\subsection{OWL Ontology based on Elements and Relationships}

The entities and relationships described above can be used to create an OWL ontology. The key parent concepts from Table 4 can be seen in OWL format in Figure 1.

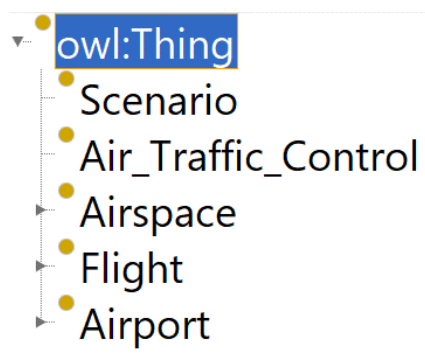

Figure 1: High-level view of scenario-based aviation ontology.

Upon expanding the high-level view from Figure 1 to see the components of the Airspace, Airport and Flight classes, the view in Figure 2 is obtained. The Flight_State class has also been expanded in Figure 2. 

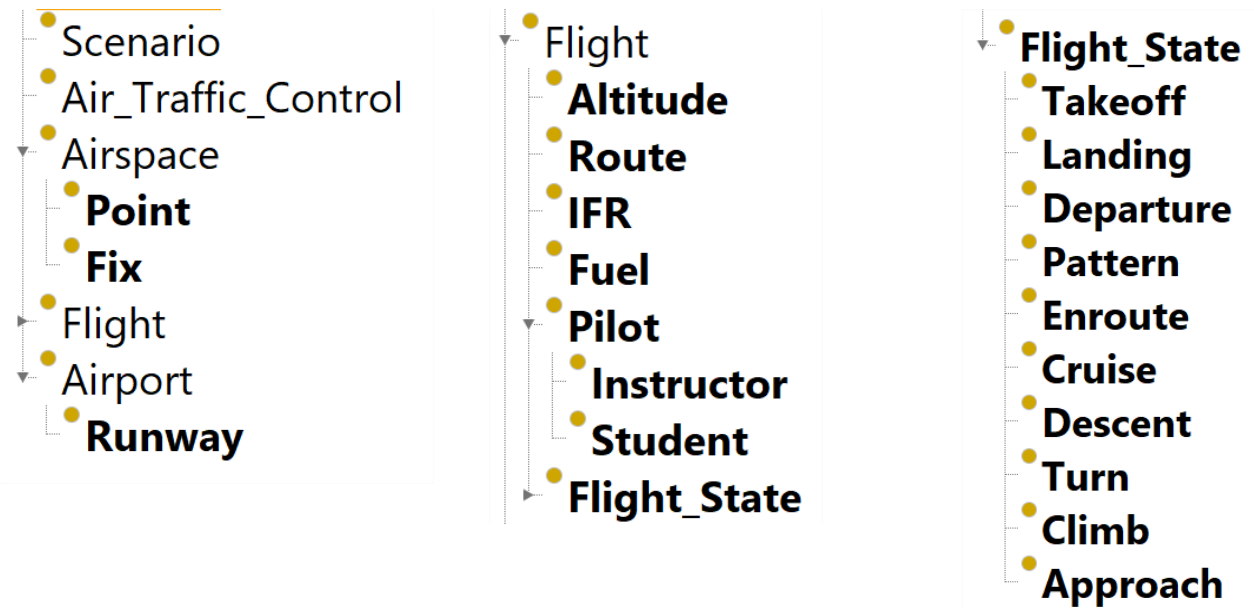

Figure 2: Components of airspace, airport and flight class in ontology.

There are very few elements described here since the threshold of repetition was set to be high and a very small set of natural-language scenarios was considered. However, the aim of the case study is to show that the elements obtained in this manner are useful and can be used to design a domain model to create scenarios of this type. In order to understand the value of this approach, this ontology will be compared to an existing aviation ontology in the next section.

\section{COMPARISON WITH EXISTING AVIATION ONTOLOGY}

\subsection{Aviation Scenario Definition Language}

First proposed by Jafer et. al. in 2016, ASDL provides a mechanism for aviation scenario generation from a model-driven perspective (Jafer, Chhaya and Durak, et al. 2016). By creating a common, standardized language for defining aviation scenarios, the duplicate effort often seen in the aviation simulation scenario generation process can be avoided. Developed in the Eclipse Modeling Framework (EMF), ASDL is a DSL which currently supports four major categories of flight operation scenarios: departure, en route, reroute, and landing, as well as air traffic management concepts such as controller-pilot communication (Jafer, Chhaya and Durak, et al. 2018) (Chhaya, et al. 2018).

The creation of ASDL and ASDL scenarios followed three major steps: (1) ASDL-specific ontology creation, (2) EMF definition and metamodel creation, and (3) design and implementation of a scenario in the instantiated model. The ASDL ontology consists mainly of key terms defined by the FAA, as well as keywords used in communication between the pilot and air traffic control (ATC). All ontology terms included in ASDL were obtained from (Order, F.A.A. 1993), (SESAR n.d.), and (Federal Aviation Administration 2012). In the interest of reusability, the ASDL ontology focuses on all aspects of a flight, including the physical aircraft as well as ATC and pilot communications and procedures. By including such aspects, the ontology can be used in all forms of aviation simulators, including ATC simulators and pilot training simulators (Jafer, Chhaya and Durak 2017).

The ASDL ontology (Jafer, Chhaya and Durak 2017) consists of 123 classes including extensions for landing, departure and en route flights (Jafer, Chhaya and Updegrove, et al. 2018). That is a much larger undertaking than the ontology created in this paper, but a comparison of the entities that are present here can show whether a scenario-driven approach works for creating ontologies for domain models.

\subsection{Comparison of elements with ASDL Ontology}

This section discusses the previously published ASDL ontology and compares it to the version generated here. The core elements of the two ontologies are shown in Figure 3. 

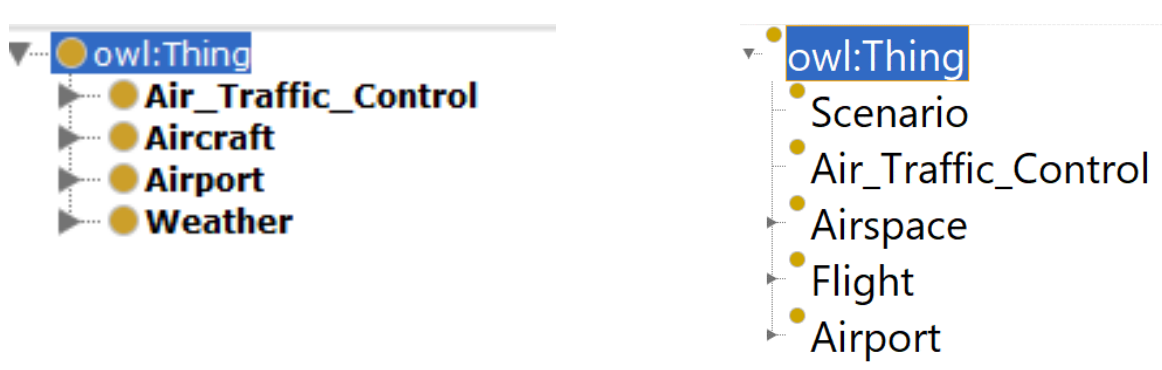

Figure 3: Comparison of high-level ontologies of ASDL (Jafer, Chhaya and Durak 2017) and scenariodriven approach.

It can be seen that weather conditions did not appear often in the scenarios chosen for this approach, so the Weather class is missing. The other classes are identical, except for the addition of a super class called "Scenario", which is not specific to the aviation domain, and the fact that an "Aircraft" is referred to as a "Flight" in the scenario-driven version.

The "Aircraft" and "Flight" classes are compared next in Figure 4. It can be seen that the properties in the original ASDL ontology are a lot more detailed. However, the items captured in the scenario-driven ontology are able to define flights from the perspective of a student pilot and instructor, even if they are lacking in the physical properties of the aircraft being simulated.
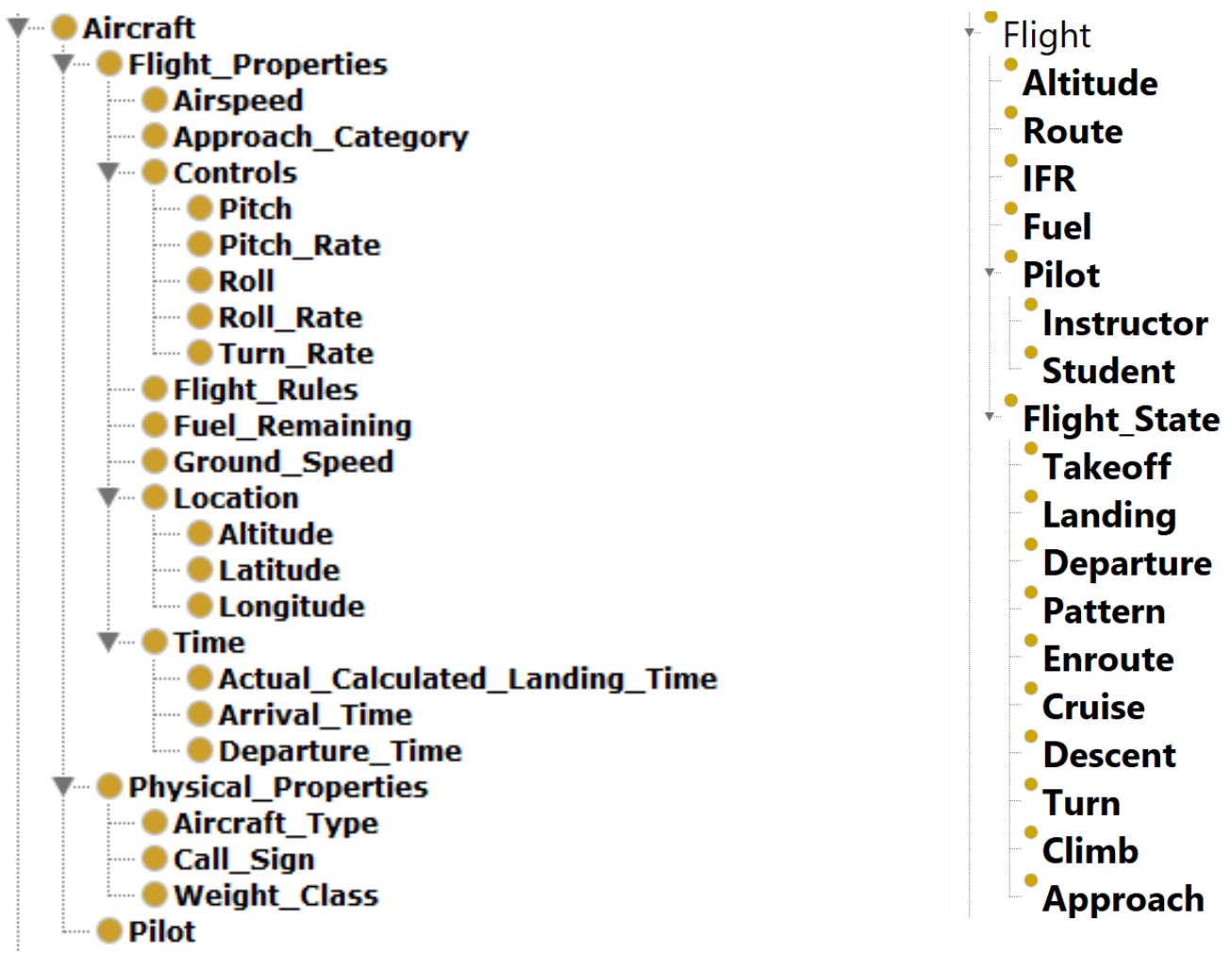

Figure 4: Comparison of aircraft and flight components in ontologies of ASDL (Jafer, Chhaya and Durak 2017) and scenario-driven approach.

It shows that a much deeper understanding of the domain was required to create the first ontology, but on the other hand, it is possible that specific flight properties and controls that have been included in the detailed ontology do not feature in the generation of a scenario for a student pilot. It is a more efficient solution when classes are only created if they are expected to be utilized instead of creating classes for 
every concept in the domain. It is also a safe assumption that given enough scenarios that need to be modeled, physical characteristics or flight properties would appear in a scenario-driven model as long as they are relevant to the scenario at hand. One drawback of this approach is that if a "unique" scenario needs to be modeled, relevant characteristics might slip by if we only account for frequency of mention.

\section{CONCLUSION AND FUTURE WORK}

Ontologies are the first step towards creating domain-specific languages (DSLs), which can increase productivity greatly if an appropriate DSL is created for the application. An ontology is a vocabulary for a domain and describes the key concepts and their relationships. Instead of studying the domain and searching for meaningful concepts, this paper approaches the ontology through natural-language scenarios in the domain. If the frequency of words in these scenarios is analyzed, key concepts become obvious as they are used repeatedly in scenarios and the ontology or domain modeling engineer only needs to investigate these specific concepts to understand the overall big picture of the domain. By knowing exactly what key words to look for, concepts that were not mentioned in the scenarios also become easier to find.

As a case study, 30 short flight training scenarios were analyzed and a small picture of the aviation domain was obtained from these scenarios. Using these key concepts, an ontology was created in the OWL format and compared with an existing aviation ontology. Several overlapping concepts were found which shows the value in looking at an ontology from this perspective.

Future work includes the generation of an aviation ontology with a much larger scope so it can be properly compared to the ASDL ontology. A large number of scenarios will need to be written or obtained for this purpose. One other concern that needs to be identified is the use of synonyms in natural-language scenarios. For instance, the terms "flight" and "aircraft" are sometimes used interchangeably and it will be a manual process to identify the "correct" word to use when creating the ontology.

A future experiment would involve the creation of a similar ontology by a user who has no domain expertise and only a set of scenarios. The ontology can be compared to one created by a domain expert and certain metrics can be used to determine the value of this approach: time taken in both cases, knowledge required and the number of errors in the ontology made by the new approach. If the approach is not justified by the experiment, there may still be potential in its use to validate an ontology after its generation through other means as it would enable quick identification of missing terms.

As natural language poses its own issues, they will need to be manually solved currently, but the time saved by automatically analyzing word frequencies and finding nouns among the most frequent words instead of studying a domain thoroughly from scratch seems to be very advantageous and should be exploited.

\section{REFERENCES}

Alatrish, Emhimed. 2013. "Comparison Some of Ontology Editors." Management Information Systems 8 (2): 018-024.

Bechhofer, Sean. 2009. "OWL: Web Ontology Language." In Encyclopedia of Database Systems, by Ling Liu and M. Tamer Özsu, 2008-2009. Boston: Springer US.

Calfior, Fred J., and Douglas W. Miller. 1994. Flights of 13MIKE. Prescott, AZ: CalMil Publishing.

Čeh, Ines, Matej Črepinšek, Tomaž Kosar, and Marjan Mernik. 2011. "Ontology driven development of domain-specific languages." Computer Science and Information Systems 2 317-342.

Chhaya, Bharvi, Shafagh Jafer, William B. Coyne, Neal C. Thigpen, and Umut Durak. 2018. "Enhancing Scenario-Centric Air Traffic Control Training." 2018 AIAA Modeling and Simulation Technologies Conference. 1399. 
Durak, Umut, Okan Topcu, Robert Siegfried, and Halit Oguztuzun. 2014. "Scenario Development: A Model-Driven Engineering Perspective." Simulation and Modeling Methodologies, Technologies and Applications (SIMULTECH), 2014 International Conference on. IEEE. 117-124.

Faure, David, Claire Nédellec, and Céline Rouveirol. 1998. Acquisition of Semantic Knowledge using Machine learning methods: The System "ASIUM". Paris: Universite Paris Sud.

Federal Aviation Administration. 2012. AFS Flight Program Flight Operations Manual. Federal Aviation Administration.

Federal Aviation Administration. 2013. Example Scenarios. $19 \quad$ March. https://www.faa.gov/training_testing/training/fits/scenarios/.

GSD Product Development Group, SISO. 2014. Guideline on Scenario Development for Simulation Environments. Orlando: Simulation Interoperability Standards Organization.

Jafer, Shafagh, Bharvi Chhaya, and Umut Durak. 2017. OWL ontology to Ecore metamodel transformation for designing a domain specific language to develop aviation scenarios. Accessed 2 5, 2019. https://elib.dlr.de/112673.

Jafer, Shafagh, Bharvi Chhaya, Jessica Updegrove, and Umut Durak. 2018. "Schema-based Ontological Representations of a Domain-Specific Scenario Modeling Language." Journal of Simulation Engineering 1.

Jafer, Shafagh, Bharvi Chhaya, Umut Durak, and Torsten Gerlach. 2018. "Automatic Generation of Flight Simulation Scenarios with Aviation Scenario Definition Language." Journal of Aerospace Information Systems 1-10.

Jafer, Shafagh, Bharvi Chhaya, Umut Durak, and Torsten Gerlach. 2016. "Formal Scenario Definition Language for Aviation: Aircraft Landing Case Study." AIAA Modeling and Simulation Technologies Conference. 3521.

Mernik, Marjan, Jan Heering, and Anthony M Sloane. 2005. "When and How to Develop Domain-Specific Languages." ACM Computing Surveys (CSUR) 37 (4): 316-344.

Order, F.A.A. 1993. "7110.65 J, Air Traffic Control." Washington, DC: Federal Aviation Administration.

Pereira, María J., João Manuel Sousa Fonseca, and Pedro Rangel Henriques. 2016. "Ontological approach for DSL development." Computer Languages, Systems \& Structures 45 (45): 35-52. Accessed 25 , 2019. https://bibliotecadigital.ipb.pt/bitstream/10198/12760/1/1-s2.0-s1477842415300270main.pdf.

2016. Protégé Home Page. http://protege.stanford.edu/.

Sen, Sagar, Benoit Baudry, and Hans Vangheluwe. 2010. "Towards Domain-specific Model Editors with Automatic Model Completion." SIMULATION 86 (2).

SESAR. n.d. EUROCONTROL ATM Lexicon. Single European Sky ATM Research.

Shamsfard, Mehrnoush, and Ahmad Abdollahzadeh Barforoush. 2004. "Learning ontologies from natural language texts." International Journal of Human-Computer Studies 17-63.

Shamsfard, Mehrnoush, and Ahmad Abdollahzadeh Barforoush. 2003. "The state of the art in ontology learning: A framework for comparison." Knowledge Engineering Review 293-316.

SISO Base Object Model Product Development Group. 2006. Base Object Model (BOM) Template. Orlando: Simulation Interoperability. 
Thibault, Scott, Renaud Marlet, and Charles Consel. 1999. "Domain-specific languages: from design to implementation application to video device drivers generation." IEEE Transactions on Software $\begin{array}{llllll}\text { Engineering } 25 & \text { (3): 363-377. Accessed } 2019 . \quad \text { http://dblp.uni- }\end{array}$ trier.de/db/journals/tse/tse25.html.

Yao, Zhong, and Quan Zhang. 2009. "Protégé-Based Ontology Knowledge Representation for MIS Courses." International Conference on Web Information Systems and Mining.

\section{AUTHOR BIOGRAPHIES}

BHARVI CHHAYA is a Ph.D. candidate in Electrical Engineering and Computer Science at EmbryRiddle Aeronautical University. She received her Master of Software Engineering and Bachelor of Science in Aerospace Engineering degrees from Embry-Riddle. Her doctoral research focuses on scenario-based domain-specific language development. Her email address is chhayab@my.erau.edu.

SHAFAGH JAFER is an Assistant Professor of Software Engineering at Embry-Riddle Aeronautical University. She holds a PhD in Computer Systems Engineering from Carleton University in Canada. Her research interest include simulation-based engineering, mission-critical software systems, and model-based development. Her email address is jafers@erau.edu. 